\title{
Apprendre à planifier ses cours: évolution, sources de changement et processus d'apprentissage
}

\section{Kim Lê Van et Jean-Louis Berger}

La rédaction de cet article a été réalisée dans le cadre d'un projet soutenu par le fonds national suisse de la recherche scientifique. "L'évolution des conceptions durant la formation à l'enseignement» (Subside N¹00019_146351; Requérant principal: Jean-Louis Berger, Institut fédéral des hautes études en formation professionnelle; Co-requérants: Marcel Crahay, Université de Genève et Carmela Aprea, Friedrich-Schiller-Universität Jena).

Planifier des cours est l'une des compétences centrales que les enseignant-e-s développent durant leur formation. Les sources et processus régissant l'apprentissage des pratiques de planification restent encore largement inexplorés. Trente entretiens semi-directifs menés auprès d'enseignants du secondaire récemment diplômés ont permis de conclure que diverses pratiques de planification étaient apprises et valorisées par les enseignant-e-s. De plus, ces pratiques relèvent de sources variables qui ne se limitent pas à la formation, ainsi que d'apprentissages de types formels, non formels et informels. Cette étude révèle ainsi comment s'articulent les facteurs influençant l'évolution des pratiques de planification.

\section{Introduction}

La planification des cours désigne «l'activité d'anticipation de l'enseignant pendant la phase pré-active, c'est-à-dire une série de processus grâce auxquels un individu se représente le futur, fait l'inventaire des fins et des moyens et construit un cadre anticipé susceptible de guider ses actions à venir» (Riff \& Durand, 1993, p. 84). Planifier comprend réflexions, prises de décision et jugements de la part de l'enseignant-e (Clark \& Yinger, 1979). La préparation des cours offre ainsi un lieu des plus favorables à une adaptation de l'enseignement aux caractéristiques des élèves, notamment par le choix des méthodes d'enseignement ou des supports de cours. Elle est l'une des compétences centrales que les enseignant-e-s développent durant leur formation, voire la compétence la plus impor- 
tante apprise au cours de la formation selon certains (Mutton, Hagger \& Burn, 2011). De ce fait, les changements de connaissances et de pratiques relatifs à la planification sont considérés comme un indicateur critique du succès de la formation à l'enseignement. L'un des objectifs fondamentaux de la formation pédagogique est de promouvoir un développement professionnel en invitant les futur-e-s enseignant-e-s à acquérir des pratiques de planification et des connaissances sur l'activité de planification. Ce développement dépend d'une multitude de facteurs s'influençant mutuellement, tels que l'enrichissement des connaissances pédagogiques générales des enseignant-e-s ${ }^{1}$ (Mutton et al., 2011), le changement de leurs croyances sur l'enseignement (Richardson \& Placier, 2001), et le renforcement de leur sentiment d'efficacité personnelle (Siwatu \& Chesnut, 2015). Au-delà de l'importance certaine de la formation à l'enseignement, les recherches ont montré que la planification des enseignant-e-s est fondée sur une pluralité d'autres expériences, lesquelles incluent l'expérience d'enseignement, l'expérience en tant qu'élève (façonnant sa représentation de l'enseignement; Grossman, 1989) ou encore l'expérience de la vie privée (Richardson \& Placier, 2001; Wanlin, 2009).

Débutant dans les années 1970 aux États-Unis d'Amérique (Clark \& Yinger, 1979; Zahorick, 1975), la recherche sur la pensée des enseignant-e-s lors de la planification de leur cours s'est, dans un premier temps, essentiellement penchée sur les processus cognitifs mis en œuvre pour concevoir les activités d'enseignement-apprentissage, les éléments auxquels les enseignant-e-s pensent en vue de planifier et les facteurs considérés dans la prise de décision (Charlier, 1989). Certaines études ont porté sur les connaissances et croyances des enseignant-e-s relatives à l'acte de planification (Clark \& Yinger, 1979; Yildirim, 2003) ainsi que sur leurs liens avec les pratiques d'enseignement effectivement mises en œuvre en classe. Par exemple, Yildirim (2003) a montré que les enseignant-e-s poursuivent divers buts dans la planification, d'ordres pédagogiques, mais aussi administratifs et personnels. Malgré une littérature abondante sur le sujet et au-delà des apports de la recherche sur la planification, force est de constater avec Mutton et al. (2011), que les connaissances sur l'apprentissage ou l'évolution des pratiques de planification au cours du temps sont lacunaires. Ainsi, le présent article rapporte les conclusions d'une étude qui visait à décrire comment évoluent, dans un échantillon d'enseignant-e-s du secondaire novices, les pratiques de planification durant la formation pédagogique ainsi que durant les premières années d'enseignement.

\section{L'évolution de la planification de l'enseignement}

L'évolution de la planification de l'enseignement chez les novices a fait l'objet de nombreux travaux dans des contextes culturels divers; en Suisse (p.ex., Vaudroz, Berger, Girardet \& Aprea, 2015, janvier; Lê Van \& Berger, 2016, septembre; Baer, Kocher, Wyss, Guldimann, Larcher \& Dörr, 2011), aux États-Unis d'Amérique (p. ex., Kagan \& Tippins, 1992), en Belgique (p. ex., Broeckmans, 
1986), en Allemagne (p. ex., Brodhäcker, 2014) ou sous forme de comparaison internationale (Blömeke et al., 2008). Globalement, les résultats de ces études convergent pour conclure à des changements conséquents se produisant non seulement durant la formation, mais aussi lors des premières années d'exercice de la profession (Broeckmans, 1986; Kagan \& Tippins, 1992). Bien que les études mobilisées fussent réalisées dans des contextes culturels et scolaires divers, les constats relatifs à la formation pédagogique et au rôle de l'expérience dans la planification sont fortement similaires. Ainsi, nous référons à ce corpus d'études pour décrire l'état des connaissances sur l'évolution de la planification de l'enseignement.

\section{Le rôle de la formation pédagogique}

Durant la formation pédagogique ${ }^{2}$, les compétences de planification, définies à partir des travaux d'Altet (2012) comme «l'ensemble des savoirs, savoir-faire, savoir-être mais aussi les faire et être» (p. 49) nécessaires à la planification des cours, se développeraient régulièrement de la première à la dernière année de formation pour atteindre un niveau proche de celui d'enseignant-e-s chevronné-e-s; la première année suivant l'entrée dans le métier serait néanmoins accompagnée d'une régression dans ces compétences (Baer et al., 2011). Les conclusions de Kagan et Tippins (1992) révèlent que les évolutions ne seraient pas identiques pour tous les enseignant-e-s en formation. Pour la majorité, les chercheurs observent une tendance à plus fortement structurer et détailler les plans de cours, ce qui vise deux objectifs: s'assurer qu'aucun contenu ne sera omis durant le cours et maintenir une discipline dans des classes d'adolescents nombreux. Pour d'autres, dont le sentiment d'efficacité personnelle est plus élevé, les changements consistent à adopter une planification moins linéaire et détaillée, autrement dit plus flexible, afin de pouvoir s'adapter à la réalité de la classe et d'intervenir de manière improvisée (Tochon, 1993). De plus, par la formation pédagogique, les objets de considération des enseignants pendant qu'ils planifient évoluent; c'est ce que révèlent des études réalisées dans divers contextes culturels (Vaudroz et al., 2015, janvier; Blömeke et al., 2008; Mutton, et al., 2011). L'importance accordée au choix du matériel et des tâches décroit alors que celle donnée aux connaissances préalables des élèves et aux méthodes didactiques s'accroit après une seule année de formation selon une étude menée en Suisse (Vaudroz et al., 2015, janvier). Le développement des connaissances sur les élèves constituerait en particulier un facteur de changement des pratiques de planification selon l'étude des perceptions d'enseignant-e-s récemment certifié-e-s en Angleterre (Mutton et al., 2011). En résumé, les études mentionnées indiquent que grâce à la formation, les enseignant-e-s présentent un bagage de connaissances accru sur la manière de planifier, qu'ils mobilisent ce bagage de manière plus large et qu'ils considèrent des objets de planification plus complexes.

Étant donné que l'évolution de la planification se poursuit indubitablement une fois la formation achevée (Kagan \& Tippins, 1992; Mutton et al., 2011), il 
est essentiel de l'explorer durant les premières années d'exercice du métier. Cette exploration revêt une pertinence pour les formations pédagogiques en ce qu'elle révèle comment les enseignant-e-s mobilisent les connaissances et pratiques développées en formation et comment ils/elles continuent de développer leurs connaissances et pratiques dans la ou les écoles où ils/elles exercent.

\section{Le rôle de L'expérience}

Il n'est plus à établir que l'apprentissage des enseignant-e-s relève tant de la formation dispensée que de l'expérience d'enseignement (Ellis \& Orchard, 2014; Simbagoye, 2008). En effet, en vue de favoriser l'enseignement, l'enseignant-e s'appuie tant sur des connaissances pédagogiques générales et des connaissances pédagogiques du contenu ${ }^{3}$, que sur des connaissances fondées sur ses expériences pour développer une manière qu'il/elle estime satisfaisante de planifier son cours (Altet (2012) parlerait de «savoirs pour enseigner»). Selon Richardson (1996), trois types d'expériences pourraient avoir un impact sur le développement des connaissances des enseignant-e-s: a) des expériences personnelles, soit liées à la vie non-professionnelle, b) des expériences relatives à l'apprentissage et l'enseignement, telles que les observations de l'enseignement réalisées en tant qu'élève et c) des expériences liées à la mobilisation de connaissances pédagogiques générales et du contenu. L'expérience d'enseignement permettrait divers apprentissages bénéfiques tels que l'élaboration d'outils de gestion de sa planification, émergeant grâce à la résolution des problèmes quotidiens (Huberman, 1983) ou des ajustements progressifs à l'action et à la réflexion pédagogique (Richardson $\&$ Placier, 2001). Cette capacité de l'enseignant-e à appréhender les événements est désignée comme "connaissances artisanales» (craft knowledge; Grimmett \& MacKinnon, 1992). Autrement dit, il s'agit de connaissances développées par l'enseignant-e même grâce à une articulation entre connaissances spécifiques à la profession et expérience en classe.

Le développement de pratiques et connaissances relève d'une diversité de types d'apprentissage plus ou moins formels qu'il s'agit d'identifier, afin d'en comprendre l'articulation. Ainsi, une distinction de trois types d'apprentissage est proposée par Cristol et Muller (2013). L'apprentissage formel désigne un apprentissage reconnu par les hautes autorités d'éducation (École, Hautes écoles). Les connaissances acquises sont celles visées par les curriculums officiels de l'enseignement. Cet apprentissage donne lieu à une validation institutionnelle. Dit simplement, il s'agit de la forme classique d'apprentissage scolaire. L'apprentissage non formel est acquis sur le lieu de travail, en parallèle de la structure institutionnelle. Il peut être développé dans le cadre d'organisations (associations, syndicats, partis politiques) ou d'un travail collaboratif entre enseignant-e-s. Cet apprentissage non formel désigne ainsi l'apprentissage effectué dans les coulisses du cadre institutionnel. Quant à l'apprentissage informel, bien que sa définition ne fasse pas l'objet d'un consensus (Cristol \& Muller, 2013), Schugurensky (2009) le décline en trois modalités de réalisation. L'apprentissage 
informel peut se réaliser par (a) la socialisation (les évènements du quotidien participant à un apprentissage permanent, qui n'est ni provoqué, ni réalisé de manière consciente); (b) un apprentissage fortuit (un apprentissage accidentel dont l'individu prend toutefois conscience par la suite) ou (c) un apprentissage autodirigé (un apprentissage dont l'enseignant est conscient et qu'il entreprend ainsi de manière intentionnelle). Autrement dit, il s'agit d'un apprentissage effectué par des développements personnels et professionnels. Bien qu'une distinction soit établie entre ces différents apprentissages et quelle que soit la perspective adoptée, les connaissances propres à une profession naissent d'un entrelacement des types d'apprentissages et non d'un cumul.

La littérature synthétisée dans cette introduction rencontre deux limites majeures. Tout d'abord, les études se concentrent avant tout sur les changements survenant dans les pratiques de planification et moins sur les processus d'apprentissage qui régissent ces changements. Le rôle de la formation enseignante dans les changements de connaissances et de pratiques ne peut que partiellement être compris si ne sont pas considérés conjointement les impacts d'autres sources d'influence, telles que l'expérience dans l'enseignement, l'aide d'un pair plus expérimenté, la préférence pour un certains style d'enseignement ou encore les souvenirs de sa propre scolarité. La seconde limite est que la période étudiée est souvent limitée au temps de formation, négligeant les années qui la suivent celle-ci, alors qu'il s'agit là d'un moment que l'on sait significatif dans le développement professionnel (Richardson \& Placier, 2001).

\section{Objectifs de l'étude et questions de recherche}

La présente étude se propose d'aller au-delà de la littérature synthétisée en décrivant comment les pratiques de planification changent durant la formation, puis au cours des premières années d'exercice du métier. Afin de montrer l'articulation entre pratiques de planification et type d'apprentissage, l'étude explore les questions de recherche suivantes:

1. Quelles pratiques sont valorisées par les enseignant-e-s et comment décrivent-ils/elles leur évolution?

2. Quelles sont les sources auxquelles ces changements sont attribués?

3. Comment ces changements ont-ils été opérés et de quels types d'apprentissages relèvent-ils selon les enseignant-e-s?

\section{Méthodologie}

\section{Participant-e-s}

Cette étude ${ }^{14}$ repose sur 30 entretiens semi-structurés d'une durée de 45 à 90 minutes, menés auprès d'enseignant-e-s ayant achevé leur formation 2 à 3 ans auparavant. Le Tableau 1 présente différentes caractéristiques des participant-e-s. 
Tableau 1.: Description des participants

\begin{tabular}{|c|c|c|c|c|c|}
\hline $\begin{array}{l}\mathrm{N}^{\circ} \text { parti- } \\
\text { cipant }\end{array}$ & \begin{tabular}{|l|}
$\begin{array}{l}\text { Branche } \\
\text { première }\end{array}$ \\
\end{tabular} & $\begin{array}{l}\text { Branche } \\
\text { seconde }\end{array}$ & Sexe & Âge & Type d'établissement \\
\hline 1 & Français & $\mathrm{n} / \mathrm{a}$ & F & 29 & Cycle d'orientation \\
\hline 2 & Physique & $\mathrm{n} / \mathrm{a}$ & $\mathrm{F}$ & 32 & ECG \\
\hline 3 & Français & Histoire & $\mathrm{H}$ & 28 & Cycle d'orientation \\
\hline 4 & Chimie & $\mathrm{n} / \mathrm{a}$ & $\mathrm{H}$ & 38 & ECG \\
\hline 5 & Géographie & $\begin{array}{l}\text { Education } \\
\text { citoyenne }\end{array}$ & $\mathrm{F}$ & 32 & Ecole de commerce \\
\hline 6 & Physique & $\begin{array}{l}\text { Mathéma- } \\
\text { tiques }\end{array}$ & $\mathrm{H}$ & 35 & Cycle d'orientation \\
\hline 7 & Allemand & $\mathrm{n} / \mathrm{a}$ & $\mathrm{F}$ & 47 & Cycle d'orientation \\
\hline 8 & Mathématiques & \begin{tabular}{|l|}
$\begin{array}{l}\text { Informa- } \\
\text { tique }\end{array}$ \\
\end{tabular} & F & 50 & ACPO / CTP \\
\hline 9 & Géographie & $\mathrm{n} / \mathrm{a}$ & $\mathrm{H}$ & 41 & Collège / Cycle d'orientation (classe relais) \\
\hline 10 & Biologie & $\mathrm{n} / \mathrm{a}$ & $\mathrm{H}$ & 30 & Cycle d'orientation \\
\hline 11 & Anglais & $\mathrm{n} / \mathrm{a}$ & $\mathrm{F}$ & 35 & Cycle d'orientation \\
\hline 12 & Français & $\mathrm{n} / \mathrm{a}$ & $\mathrm{F}$ & 37 & Cycle d'orientation \\
\hline 13 & Anglais & $\mathrm{n} / \mathrm{a}$ & $\mathrm{F}$ & 45 & ECG \\
\hline 14 & Histoire & Anglais & $\mathrm{H}$ & 31 & Collège / Collège pour adultes \\
\hline 15 & Histoire & Géographie & $\mathrm{H}$ & 29 & Collège/ACPO \\
\hline 16 & Mathématiques & $\mathrm{n} / \mathrm{a}$ & $\mathrm{F}$ & 27 & CFPT \\
\hline 17 & Mathématiques & Economie & $\mathrm{H}$ & 36 & Cycle d'orientation \\
\hline 18 & Biologie & $\mathrm{n} / \mathrm{a}$ & $\mathrm{H}$ & 38 & Collège \\
\hline 19 & Chimie & $\mathrm{n} / \mathrm{a}$ & $\mathrm{H}$ & 33 & ECG \\
\hline 20 & Géographie & $\mathrm{n} / \mathrm{a}$ & $\mathrm{F}$ & 28 & Cycle d'orientation /Collège pour adultes \\
\hline 21 & Géographie & $\begin{array}{l}\text { Education } \\
\text { citoyenne }\end{array}$ & $\mathrm{H}$ & 39 & Cycle d'orientation \\
\hline 22 & Français & $\mathrm{n} / \mathrm{a}$ & $\mathrm{F}$ & 31 & Cycle d'orientation \\
\hline 23 & Français & $\mathrm{n} / \mathrm{a}$ & $\mathrm{F}$ & 41 & Cycle d'orientation \\
\hline 24 & Allemand & $\mathrm{n} / \mathrm{a}$ & $\mathrm{F}$ & 40 & Cycle d'orientation \\
\hline 25 & Allemand & Psychologie & $\mathrm{F}$ & 39 & Collège \\
\hline 26 & Géographie & $\begin{array}{l}\text { Education } \\
\text { citoyenne }\end{array}$ & F & 31 & Cycle d'orientation \\
\hline 27 & Géographie & $\begin{array}{l}\text { Education } \\
\text { citoyenne }\end{array}$ & $\mathrm{H}$ & 36 & Cycle d'orientation \\
\hline 28 & Géographie & Histoire & $\mathrm{F}$ & 38 & Cycle d'orientation \\
\hline 29 & Mathématiques & $\mathrm{n} / \mathrm{a}$ & $\mathrm{H}$ & 40 & Collège \\
\hline 30 & Physique & $\mathrm{n} / \mathrm{a}$ & $\mathrm{H}$ & 40 & Cycle d'orientation \\
\hline
\end{tabular}

Note. $\mathrm{ECG}=$ Ecole de Culture Générale $; \mathrm{ACPO}=$ Accueil du Postobligatoire $; \mathrm{CTP}=$ Centre de la Transition Professionnelle; CFPT $=$ Centre de Formation Professionnelle Technique. 
L'échantillon est composé de 16 femmes et 14 hommes, d'une moyenne d'âge de 35 ans, enseignant dans le canton de Genève. Enseignant-e-s de disciplines diverses de degré secondaire I et II en formation professionnelle ou générale, tou-te-s ont suivi une formation de 1 à 3 ans ${ }^{25}$ à l'Institut Universitaire de Formation des Enseignants (IUFE) à Genève.

Les conditions d'entrée en formation constituent, pour la plupart, des études universitaires dans les matières enseignées. La formation inclut des cours présentiels, des stages dans l'enseignement et des visites de formateurs dans les classes lorsque l'étudiant enseigne à titre de stagiaire. Elle inclut des ateliers de planification didactique qui visent le développement de diverses compétences en matière de planification des cours. Son référentiel de compétence précise que l'enseignant-e en formation doit être progressivement apte à «transposer ses propres connaissances et ses savoir-faire dans des situations favorisant chez les élèves un apprentissage actif et progressivement autonome», à «évaluer la progression des élèves et finalement leurs capacités, leurs prestations et leurs connaissances», et enfin à «utiliser différents supports d'enseignement» (Institut Universitaire de Formation des Enseignants [IUFE], 2015).

\section{Instruments}

Le protocole d'entretien inclut vingt questions organisées en cinq thématiques: 1) le parcours de formation, 2) l'évolution générale des pratiques d'enseignement, 3) l'évolution des pratiques de gestion de classe, 4) l'évolution des pratiques de planification de cours, et 5) les représentations de l'enseignement et de l'enseignant-e. La motivation à devenir enseignant-e, le sentiment d'efficacité personnelle, ainsi que la perception de la formation sont des dimensions transversales également explorées par le protocole.

La présente étude, de par son objet, concentre ses analyses sur la partie de l'entretien consacrée à l'évolution de la planification des cours. Celle-ci inclut spécifiquement des questions relatives aux pratiques de planification passées et présentes, ainsi que des questions sur la place de la formation, de l'expérience en classe ou d'autres éléments intervenant dans l'évolution des pratiques, ou encore les conseils qui seraient donnés à un-e nouvel-le enseignant-e en matière de planification (voir Annexe 1). Sur accord des participant-e-s, les entretiens ont été enregistrés en audio et intégralement retranscrits selon un mode verbatim. Les enseignant-e-s interrogé-e-s ont tou-te-s été assuré-e-s, par écrit, de l'anonymat des données et du respect de leur intégrité. La participation était volontaire et non rémunérée. Les participant-e-s pouvaient interrompre l'entretien à tout moment.

\section{Analyse des données}

Les transcriptions ont été importées dans le logiciel de codage NVivo10 (QSR International, 2012). L'analyse des données a suivi un processus itératif d'analyse de contenu et de développement d'un système de codage, inspiré de la littérature 
sur l'impact de la formation sur l'évolution des pratiques d'enseignement. Le codage a été élaboré à partir d'une précédente étude (Lê Van \& Berger, 2016, septembre). Les pratiques de planification ont été regroupées en six catégories thématiques, réunissant des codes relatifs, selon les recommandations de Saldaña (2013).

Le Tableau 2 présente un aperçu des catégories et des codes élaborés.

\section{Tableau 2: Description des codes attribués à la planification des cours}

\begin{tabular}{|c|c|c|c|}
\hline $\begin{array}{l}\text { Catégorie } \\
\text { thématique }\end{array}$ & Codes & Description & $\begin{array}{l}\text { Question(s) apportant } \\
\text { des éléments de réponse }\end{array}$ \\
\hline $\begin{array}{l}\text { Considérations } \\
\text { dans la planifi- } \\
\text { cation }\end{array}$ & $\begin{array}{l}\text { Didactique } \\
\text { Conditions du groupe } \\
\text { d'apprentissage } \\
\text { Prescriptions institution- } \\
\text { nelles } \\
\text { Contingences } \\
\text { Partage et équivalence } \\
\text { entre enseignants } \\
\text { Spécificités de la disci- } \\
\text { pline } \\
\text { Passé en tant qu'élève }\end{array}$ & $\begin{array}{l}\text { Les éléments que les } \\
\text { enseignants prennent en } \\
\text { considérations lorsqu'ils } \\
\text { planifient leurs cours. }\end{array}$ & $\begin{array}{l}\text { Toutes les questions } \\
\text { comprises dans la partie } \\
\text { "planification» du guide } \\
\text { d'entretien. }\end{array}$ \\
\hline $\begin{array}{l}\text { Pratiques de } \\
\text { planification }\end{array}$ & $\begin{array}{l}\text { Objectifs } \\
\text { Activités } \\
\text { Forme du plan }\end{array}$ & $\begin{array}{l}\text { La forme pratique que } \\
\text { prend la planification des } \\
\text { cours. }\end{array}$ & $\begin{array}{l}\text { En ce qui vous concerne, } \\
\text { comment planifiez-vous } \\
\text { vos cours? } \\
\text { Quelle place donnez-vous } \\
\text { à la planification dans } \\
\text { votre travail? }\end{array}$ \\
\hline $\begin{array}{l}\text { Sources de la } \\
\text { pratique de plani- } \\
\text { fication }\end{array}$ & $\begin{array}{l}\text { Formation } \\
\text { Autre significatif } \\
\text { Facteurs personnels }\end{array}$ & $\begin{array}{l}\text { Les éléments à l'origine } \\
\text { de la pratique de planifi- } \\
\text { cation. }\end{array}$ & $\begin{array}{l}\text { Est-ce que vous voyez } \\
\text { des éléments particuliers } \\
\text { qui auraient joué un rôle } \\
\text { dans cette évolution de } \\
\text { pratique? } \\
\text { Est-ce que vous avez } \\
\text { le sentiment que la } \\
\text { formation a joué un rôle } \\
\text { dans cette évolution? }\end{array}$ \\
\hline $\begin{array}{l}\text { Conséquences de la } \\
\text { pratique de plani- } \\
\text { fication }\end{array}$ & $\begin{array}{l}\text { Conséquences sur les } \\
\text { élèves } \\
\text { Conséquences sur l'ensei- } \\
\text { gnant }\end{array}$ & $\begin{array}{l}\text { Les conséquences } \\
\text { observées de l'application } \\
\text { de certaines pratiques de } \\
\text { planification. }\end{array}$ & $\begin{array}{l}\text { Toutes les questions } \\
\text { comprises dans la partie } \\
\text { "planification" du guide } \\
\text { d'entretien. }\end{array}$ \\
\hline $\begin{array}{l}\text { Perception de la } \\
\text { formation }\end{array}$ & $\begin{array}{l}\text { Perception de la } \\
\text { formation }\end{array}$ & $\begin{array}{l}\text { La vision que les parti- } \\
\text { cipants ont de la qualité } \\
\text { et des caractéristiques de } \\
\text { la formation à l'ensei- } \\
\text { gnement suivie. }\end{array}$ & $\begin{array}{l}\text { Est-ce que vous avez } \\
\text { le sentiment que la } \\
\text { formation a joué un rôle } \\
\text { dans cette évolution? }\end{array}$ \\
\hline $\begin{array}{l}\text { Le Moi-Planifi- } \\
\text { cateur }\end{array}$ & $\begin{array}{l}\text { Sentiment d'efficacité } \\
\text { personnelle } \\
\text { Coût et valeur } \\
\text { Personnalité }\end{array}$ & $\begin{array}{l}\text { La perception de sa } \\
\text { propre planification. }\end{array}$ & $\begin{array}{l}\text { Aujourd'hui, avez-vous } \\
\text { le sentiment d'avoir } \\
\text { adopté une méthode de } \\
\text { travail pour planifier vos } \\
\text { cours qui répond à vos } \\
\text { objectifs? } \\
\text { Est-ce que cette planifi- } \\
\text { cation nécessite beaucoup } \\
\text { d'investissement? }\end{array}$ \\
\hline
\end{tabular}




\begin{tabular}{|c|c|c|c|}
\hline $\begin{array}{l}\text { Pratiques } \\
\text { valorisées }\end{array}$ & $\begin{array}{l}\text { Structure de la planifi- } \\
\text { cation } \\
\text { Didactique } \\
\text { Régulation } \\
\text { Exploitation du } \\
\text { programme } \\
\text { Collaboration }\end{array}$ & $\begin{array}{l}\text { Pratiques et conceptions } \\
\text { codées par thèmes }\end{array}$ & $\begin{array}{l}\text { Imaginez que vous devez } \\
\text { conseiller un enseignant } \\
\text { qui doit planifier un } \\
\text { cours dans votre sujet } \\
\text { d'expertise pour la toute } \\
\text { première fois. Quels } \\
\text { conseils en matière de } \\
\text { planification lui donne- } \\
\text { riez-vous? } \\
\text { Aujourd'hui, avez-vous } \\
\text { le sentiment d'avoir } \\
\text { adopté une méthode de } \\
\text { travail pour planifier vos } \\
\text { cours qui répond à vos } \\
\text { objectifs? }\end{array}$ \\
\hline $\begin{array}{l}\text { Type d'appren- } \\
\text { tissage }\end{array}$ & $\begin{array}{l}\text { Formel } \\
\text { Non-formel } \\
\text { Informel }\end{array}$ & $\begin{array}{l}\text { Le type d'apprentissage } \\
\text { ayant mené à une } \\
\text { pratique de planification }\end{array}$ & $\begin{array}{l}\text { Toutes les questions } \\
\text { comprises dans la partie } \\
\text { "planification» du guide } \\
\text { d'entretien. }\end{array}$ \\
\hline
\end{tabular}

Ce tableau offre à voir les codes créées lors de l'analyse des entretiens, leur description, leur regroupement en catégories thématiques parmi 106 instances traitant de la planification des cours., ainsi que les questions ayant permis d'explorer les dimensions visées. L'analyse des données s'est faite en deux temps. C'est tout d'abord un processus itératif qui a été appliqué, entre (i) la création et la définition de codes et (ii) une analyse de contenu. Celui-ci s'est basé sur les réponses directes aux questions explorant les considérations relatives à la planification, les pratiques, les sources de changement de pratiques, les conséquences de l'évolution des pratiques en question, la perception de la formation et enfin le «moi-planificateur». Â défaut d'un accès direct aux pratiques effectives de planification que la présente démarche méthodologique n’est pas en mesure d'offrir, les pratiques mobilisées reposent sur un discours rapporté. Ces pratiques rapportées sont issues de réponses à des questions telles que «de quelle manière planifiez-vous vos cours à vos débuts dans l'enseignement/aujourd'hui?» ou "pourquoi ce changement?», permettant d'identifier les pratiques mobilisées. Dans un second temps, sur la base d'une démarche déductive, les types d'apprentissages formel, informel et non formel (Cristol \& Muller, 2013) ont été attribués aux pratiques abordées.

Des accords inter-juges ont été réalisés sur un échantillon aléatoire de 30 à 35 unités de sens, sur les groupes de pratiques de planification, les sources d'évolution de pratique ${ }^{36}$ et sur les types d'apprentissage. Les accords, mesurés par le kappa de Cohen, sont satisfaisants. ${ }^{7}$

Cette étude visant l'exploration l'évolution des pratiques de planification et l'apprentissage dont elles sont issues, les résultats se basent ici sur les pratiques rapportées, les sources d'un changement de pratiques, ainsi que le type d'apprentissage (formel, informel ou non-formel). 


\section{Résultats}

Les sources de changement dans les pratiques de planification des cours: entre formation, "autres significatifs" et facteurs personnels

À la question "voyez-vous des éléments particuliers qui auraient joué un rôle dans l'évolution de vos pratiques de planification?», les réponses des enseignant-e-s interrogés se groupent sous trois thèmes. (i) La formation à l'enseignement apparaît comme source essentielle de changement chez certain-e-s enseignant-e-s ${ }^{8}$. L'impact des cours, des formateurs et des travaux de groupe est notoire dans l'apprentissage de techniques de planification, entraînant ainsi un changement par rapport aux pratiques initialement mobilisées en début de formation (ou au préalable). (ii) Ce que nous avons identifié comme des «autres significatifs» a joué un rôle primordial dans l'évolution des pratiques de planification. Les interactions avec les collègues, avec les mentors ou encore avec les élèves apparaissent comme source déterminante du changement de pratiques de planification. (iii) Enfin, les facteurs personnels présentent également une influence importante. Ils désignent les éléments d'influence trouvant leur source au sein de l'enseignant-e même: divers types d'expériences personnelles et professionnelles, des souvenirs en tant qu'élève, une volonté d'améliorer son rapport aux élèves, ou la recherche d'un confort personnel.

\section{Les groupes de pratiques identifiés}

Les enseignant-e-s interrogé-e-s rapportent une diversité de pratiques mobilisées dans la planification des cours. L'examen des réponses à la question "comment planifiez-vous vos cours?» permet cinq regroupements thématiques portant sur: (a) la structuration de la planification (p. ex. construire une séquence, planifier sur le court et long terme, gérer le calendrier), (b) la pédagogie (p. ex. installer des rituels au sein de la classe, évaluer la juste quantité d'activités à planifier, varier le cours), (c) la collaboration (p. ex. assurer une équivalence du programme entre enseignant-e-s, développer des référents communs entre collègues, s'ajuster au programme de l'établissement), (d) l'exploitation du programme (p. ex. connaître le plan d'étude, appliquer ses connaissances au terrain), (e) la régulation (p. ex. savoir ajuster ou améliorer ses cours au fil des années). La présentation des résultats se concentrera sur trois groupes de pratiques (structuration, collaboration et régulation) permettant de tracer des formes d'articulation entre pratiques-sources-types d'apprentissage. ${ }^{9}$ Elle montrera également que la réflexivité apparaît comme pratique transversale et participe au développement de pratiques en matière de planification.

\section{Pratiques de structuration}

La structure, dans un sens commun, désigne «un ensemble organisé d'éléments interdépendants dont les relations obéissent à des lois spécifiques» (Raynal \& 
Rieunier, 1997, p. 350). La structure de la planification vise ainsi tant à traduire la manière d'atteindre les objectifs visés qu'à refléter une stratégie d'enseignement adaptée à la classe. Dans le discours des enseignant-e-s interrogé-e-s, la structuration évolue avec le temps vers une articulation de leur planification sur un court et long terme, une meilleure gestion du calendrier et l'identification de la manière adéquate de détailler sa planification. Ce discours fait ainsi écho à la littérature (Kagan \& Tippins, 1992). Les analyses indiquent que ces pratiques naissent d'une contribution de l'ensemble des sources et types d'apprentissage. Elles reposent tant sur l'apprentissage de la construction de séquences (acquis en formation, au travers d'un apprentissage formel), que sur l'observation ou les échanges entre collègues (acquis grâce à d'autres significatifs, au travers d'un apprentissage non formel) et sur une appropriation de la planification (acquise par des facteurs personnels, au travers d'un apprentissage informel).

L'extrait suivant souligne la contribution de la formation et de l'expérience, conjointement, à l'élaboration des pratiques de planification actuelles relatives à sa structure:

Aujourd'hui je prépare une séquence beaucoup plus facilement qu'il y a 3 ou 4 ans. [...] Je ne faisais même pas des séquences, je faisais des cours au fur à mesure. Mais maintenant je fais la séquence complète, j'ai une vision beaucoup plus globale de la séquence, que j'enrichis au fur et à mesure. [...] C'est lié au fait, déjà effectivement de l'expérience mais aussi des apports de la formation de l'enseignement $\left(\mathrm{n}^{\circ} 21\right.$, enseignant de géographie au cycle d'orientation, 39 ans).

Cet enseignant évoque l'absence de séquences dans sa planification à ses débuts. Le développement de la construction de séquences comme pratique de planification est venue plus tard, par la formation d'une part, mais également par l'expérience qui a permis une vision sur le long terme.

Bien que les différentes sources et types d'apprentissage participent de concert au développement des pratiques liées à la structuration, ils interviennent à des étapes différentes de l'évolution des pratiques de planification. La formation va permettre d'apprendre l'aspect formel d'une séquence, tout comme le travail au sein de groupes d'enseignant-e-s sera l'occasion de parfaire son programme et de réaliser par exemple l'importance de planifier sur le long terme.

Pratiques de collaboration

Marcel, Dupriez et Périsset Bagnoud (2007) caractérisent la collaboration comme "la communication entre les enseignants concernés. [...] [Elle] est présente chaque fois que plusieurs enseignants travaillent ensemble à la poursuite d'un objectif et d'un projet commun, même s'ils assumeront individuellement leurs tâches face aux élèves» (p. 10). Dans le discours des enseignant-e-s interrogé-e-s, la collaboration se réalise lorsqu'il s'agit d'assurer une équivalence entre enseignant-e-s du même établissement, de développer des référents communs, 
ou encore de répondre aux exigences d'un programme commun (programme de l'établissement, cohérence du programme entre les degrés scolaires, évaluations communes). À la différence des pratiques de structuration, celles de collaboration évoluent certes par le biais de la formation, d'autres significatifs et de facteurs personnels, mais apparaissent comme ne relevant que d'un unique type d'apprentissage: l'apprentissage non formel. En effet, il s'agit là de pratiques développées exclusivement en coulisse de la formation ou de la pratique de l'enseignement. Un enseignant souligne l'apport notable de la formation qui se fait en deçà des cours et apprentissages formels:

La formation m'a en tout cas apporté une bonne chose, c'est les collègues avec lesquels on travaille. Parce qu'après [la formation], on est tous lâchés dans d'autres établissements, on se revoit plus, mais on a quand même eu une formation ensemble. Donc, on arrive quand même durant ces deux, trois ans de formation à s'échanger, à discuter du matériel qu'on utilise [...]. Donc, il y a une sorte de formation qui se fait en parallèle de la formation. C'est celle qu'on a entre collègues, entre étudiants en formation, qui est très enrichissante également ( $\mathrm{n}^{\circ} 3$, enseignant de Français et d'Histoire au cycle d'orientation, 28 ans).

La formation est certes présentée comme source notoire dans l'acquisition de pratiques de planification, mais en ce qu'elle fournit une occasion à un apprentissage non formel, au travers de la collaboration avec des pairs.

Bien que l'aptitude à travailler en équipe soit un objectif officiel de la formation (IUFE, 2015), la mobilisation des pairs comme ressource se déroule en parallèle. La dimension collective de l'enseignement, manifestée par l'appartenance à des communautés de pratiques formées dans un premier temps par des enseignants en formation puis par des enseignants d'une certaine branche, constitue ainsi une ressource notable à l'évolution des pratiques (Russ, Sherin, \& Gamoran Sherin, 2016).

\section{Pratiques de régulation}

La régulation se définit comme le "processus qui permet à un système de se maintenant en état d'équilibre. [...] En pédagogie, l'évaluation formative est un instrument de régulation des apprentissages» (Raynal \& Rieunier, 1997, pp. 317-318). La régulation fait référence à des réflexions propres, des sentiments et actions qui sont planifiés et adaptés de manière cyclique dans le but d'atteindre certains buts personnels. L'individu tire alors bénéficie de son expérience pour la mettre à profit dans les actions à venir.

Dans le discours des enseignant-e-s interrogé-e-s, la régulation se réalise par l'ajustement de sa planification au fil du temps, l'amélioration progressive de son cours ou encore l'adaptation de son programme aux besoins et impératifs divers. Les analyses indiquent que les pratiques de régulation naissent d'une source de changement et d'un type d'apprentissage uniques, essentiellement liés à l'expé- 
rience en classe: les facteurs personnels et un apprentissage informel. Autrement dit, le perfectionnement de sa planification est dû à un processus de régulation essentiellement personnel, dont l'apprentissage n'est attribué ni à la formation ni au contact avec autrui.

Aucune des unités de sens se rapportant à la régulation, pratique pourtant jugée centrale par les enseignant-e-s interrogé-e-s, ne mentionne la formation comme étant à l'origine des pratiques s'y rapportant. Il est question d'expérience en classe, de démarche dite par tâtonnement (p. ex. tenter l'application de nouvelles pratiques de planification et leur ajustement selon leur efficacité) ou de facteurs personnels. Il ne s'agit pas là d'un apprentissage formel qui serait officiel au sein de la formation ou des plans d'étude, mais qui se développerait essentiellement par des facteurs personnels (personnalité, expérience, essai-erreur) et par des apprentissages informels. L'extrait suivant illustre l'importance de l'expérience d'enseignement, seule source d'apprentissage reconnue du processus de régulation menant à l'amélioration de sa planification.

Je pense c'est vraiment l'expérience année après année... De se rendre compte comment ça évolue, comment on a géré une année. Et puis se dire: «Ben, comment on va faire pour l'année prochaine?» Ouais, peut-être, c'est plutôt ça, se dire «j'ai peut-être passé pas assez de temps sur tel sujet parce que j'en avais passé trop sur celui d'avant. Il faudrait que l'année prochaine je fasse attention à limiter le temps» ( ${ }^{\circ} 16$, enseignante de mathématiques au Centre de Formation Professionnelle Technique, 27 ans).

Cette enseignante rapporte l'importance de l'expérience, seule source de changement dans le processus de régulation de sa planification.

Reconnaissons que le développement professionnel et le perfectionnement continu sont des objectifs annoncés par la formation (IUFE, 2015). La formation à la régulation est délicate à officialiser institutionnellement et se présente plutôt comme apprentissage transversal au cours de la formation. Ainsi, le propos est ici non pas de dénoncer une absence de sensibilité à la régulation en formation, mais bien de souligner la perception des enseignants sur la source de changement de leurs pratiques de planification. En conclusion, les pratiques rapportées par les enseignant-e-s, liées à une régulation de leur planification, sont selon elles ou eux acquis essentiellement par l'expérience.

\section{La pratique réflexive comme pratique transversale}

La réflexivité, autrement dit le processus cognitif visant à réfléchir sur sa pratique, est valorisée et présentée comme déterminante dans tout changement de pratiques de planification. Selon Perrenoud (2001), «il faut distinguer la posture réflexive du professionnel de la réflexion épisodique de chacun sur ce qu'il fait. Pour aller vers une véritable pratique réflexive, il faut que cette posture devienne quasi permanente, s'inscrive dans un rapport analytique à l'action qui devienne relativement indépendant des obstacles rencontrés ou des déceptions» 
(pp. 14-15). L'auteur ajoute qu'une pratique réflexive favorise «'accumulation de savoirs d'expérience» (p. 46). Il rend attentif au fait que toute expérience ne constitue pas un apprentissage d'office. Certaines expériences confirmeront tout au mieux l'application de pratiques satisfaisantes, instaurant une routine.

Mener une réflexion critique sur son propre travail est une pratique transversale visée dans le référentiel de compétences de la formation (IUFE, 2015), que devrait développer l'enseignant-e. Perrenoud (2001) cible l'intérêt d'une pratique réflexive dans la planification: l'optimisation de l'action passe par une préparation comprenant les différents facteurs intervenant (élèves, école, espaces de travail, collègues, ressources). La pratique réflexive sur l'action pousse fréquemment l'enseignant-e à identifier des lacunes dans sa préparation ou son anticipation. Ces dites lacunes, une fois identifiées, peuvent alors être améliorées et participer à une évolution des pratiques de planification.

Dans le discours des enseignant-e-s interrogé-e-s, la réflexivité passe par la capacité d'improviser, être flexible, savoir s'ajuster aux élèves ou encore évaluer son propre travail. Les analyses indiquent que la formation à l'enseignement a participé de manière significative à faire naître ces pratiques réflexives chez l'enseignant-e. Toutefois, la manière appropriée d'appliquer ces pratiques réflexives semble acquise par la suite, en dehors de la formation:

Mais c'est pas vraiment qu'on m'a appris quelque chose, mais qu'on m'a forcé à moi me trouver mes règles et du coup ça c'est bien quand même. [...] C'est deux ans où on nous pousse à nous poser des questions et donc, je pense que les réponses sont surtout venues de moi ou de gens que j'ai interrogés, beaucoup plus que de mes enseignants [formateurs de l'IUFE] ( $\mathrm{n}^{\circ} 14$, enseignant d'histoire et d'anglais au collège ${ }^{10}, 31$ ans).

Cet enseignant trace l'évolution entre les premiers développements d'une pratique réflexive, son élaboration et sa mobilisation. Il précise que ce n'est pas le contenu même des cours sur la planification qu'il peut mettre à profit, mais plutôt cette démarche réflexive. Ensuite, sur le lieu de travail, après la formation, ce sont les échanges entre pairs et les réflexions personnelles qui ont constitué des éléments déclencheurs d'un changement de pratiques de planification. La pratique réflexive en tant qu'outil pour un développement professionnel serait acquise en formation. Elle serait ensuite concrétisée par la suite au travers de facteurs personnels ou d'autres significatifs, sources permettant de développer ses pratiques planificatrices de manière effective. Le développement de ce processus réflexif repose donc sur différentes sources agissant à différents stades de son évolution. Il relève, par extension, de différents types d'apprentissage, étant formellement promu et inculqué par la formation (apprentissage formel) et développé tant par des dynamiques d'établissement (apprentissage non formel) que par l'expérience (apprentissage informel). 


\section{Discussion générale}

La présente étude a eu pour objectif une meilleure compréhension des pratiques de planification mobilisées par des enseignant-e-s dans leurs premières années d'exercice, ainsi que leur évolution pendant et suite à la formation. L'accès aux sources et types d'apprentissage dont relèvent lesdites pratiques offre une ouverture sur leur évolution et les multiples ressources permettant une application adaptée de pratiques de planification. En effet, l'expérience ou les échanges entre collègues se mêlent aux apports de la formation pour promouvoir l'évolution de pratiques de planification. À la jonction entre apprentissage formel, non formel et informel, l'évolution des pratiques de planification se déroule pendant et après la formation à l'enseignement, comme d'autres l'avaient déjà souligné (Vaudroz et al., 2015, janvier; Mutton et al., 2011). Les échanges entre pairs ainsi que les facteurs personnels constituent des sources notables de changement, au même titre que la formation.

Quels aspects des différentes pratiques de planification sont développés au différents stades de la formation? Les résultats indiquent qu'il serait réducteur de penser l'évolution de pratiques de planification en des associations rigides d'une pratique-une source de changement de pratique-un type d'apprentissage. Nous voyons ainsi de quelle manière l'entrelacement se réalise entre les différentes sources et types d'apprentissages, au travers d'une évolution mobilisant une diversité de ressources à différentes étapes des débuts dans la carrière enseignante.

Les pratiques de planification mobilisées par les enseignant-e-s couvrent cinq thématiques, dont trois ont été traitées afin de donner à voir des articulations diverses. L'analyse des entretiens révèle une valorisation de la structuration de la planification, réunissant des pratiques de construction de séquence, de degrés de détail et de clarté du plan, une vision sur le court et long terme ou encore de la gestion du calendrier. Cette structuration constitue l'un des aspects classiquement traité par la littérature sur le sujet (Charlier, 1989; Kagan \& Tippins, 1992; Wanlin, 2009) et son importance dans le discours des enseignant-e-s n'est ainsi en rien surprenant. Au contraire, concevoir la collaboration entre collègues enseignant-e-s à la fois comme ressource et comme nécessité dans le développement d'une planification adaptée, souligne un caractère plus discret de la planification: sa dimension collective. En effet, une planification adéquate ou pertinente semble, pour certain-e-s enseignant-e-s, passer par le développement de référents communs entre collègues ainsi que l'équivalence du programme entre ceux-ci. Ceci rejoint les buts pédagogiques et organisationnels décrits par Yildirim (2003) en les élargissant à la notion de référents communs. La construction de séquences s'apprendrait en formation, alors que leurs ajustements se feraient nécessairement avec le temps, par l'expérience d'enseignement. La planification ne se conçoit ni ne se prépare sans mise en accord avec un programme. De plus, elle se doit d'assurer une cohérence entre les classes et les 
années scolaires. Enfin, l'emphase sur des pratiques liées à la régulation dénote la centralité de l'ajustement de la planification pour une auto-amélioration au fil des années (Mutton et al., 2011). Les pratiques valorisées dans la planification des cours incluent des dimensions formelles et d'expérience qui méritent d'être mises en lumières pour leur contribution à une dimension discrète mais néanmoins centrale au développement de l'enseignant enseignant.

Passant par un apprentissage formel, non formel ou informel, certaines pratiques ne s'apprennent, selon les enseignant-e-s, que de manière non formelle pour certaines, informelles pour d'autres. Les enseignant-e-s rapportent que les pratiques de régulation ne se développent que par l'expérience propre du terrain et ne relèvent que d'un apprentissage informel (Simbagoye, 2008). La valorisation de la régulation donne ainsi à voir l'importance accordée par les enseignant-e-s à l'apprentissage informel dans l'évolution des pratiques de planification.

Les conclusions de cette étude souffrent de certaines limites relatives à la méthodologie, à l'échantillon et aux conditions diverses d'exercice du métier. Tout d'abord, la grande diversité des parcours qu'ont rencontrés les participant-e-s rend la généralisation délicate. Â cela s'ajoute la diversité des disciplines enseignées dans l'échantillon. La didactique suggérant une approche et des techniques d'enseignement propres à la matière enseignée, il est à penser que les pratiques développées et les types d'apprentissages mobilisés soient définis différemment. Cependant, les pratiques mentionnées peuvent également être conçues comme étant transversales. De même, il est possible que l'enseignement au Secondaire I ou II implique des considérations et pratiques planificatrices différentes (Kagan \& Tippins, 1992), bien qu'une distinction propre au degré d'enseignement ne soit pas ressortie dans les analyses. La méthode présente pour limite le caractère auto-rapporté des pratiques passées et actuelles. Il s'agit de ce que les enseignant-e-s perçoivent comme appris en formation ou sur le terrain et de la façon dont ils attribuent leurs changements dans la planification. En effet, le souvenir des débuts dans la profession peuvent se trouver altérés. Enfin, il apparait que les conditions d'enseignement tiennent un rôle, selon les participant-e-s, dans les pratiques de planification. Il est en effet ressorti des entretiens que l'introduction dans certains établissements de technologies telles que des tableaux interactifs entraîne modifications des pratiques de planification.

Les savoirs formels et savoirs d'expérience contribuent de concert à l'élaboration de pratiques de planification des cours. Les enseignant-e-s suivent une formation incluant des savoirs spécifiques sur la matière d'enseignement et les pratiques, qui sont ensuite affinés, appropriés et développés au travers de l'expérience in situ, en classe et au sein de l'établissement.

Cette étude met en lumière non seulement diverses pratiques de planification de cours qui évoluent, mais également, et surtout, la combinaison des facteurs qui régissent cette évolution, donnant à voir la nature complexe des changements en vigueur. 


\section{Notes}

1 Les connaissances pédagogiques générales, transcendant les disciplines scolaires, concernent l'enseignement, l'apprentissage et les apprenants. Elles intègrent notamment les connaissances des enseignants relatives à la gestion de la classe, aux stratégies d'enseignement ainsi qu'aux apprenants (Shulman, 1987).

2 Notons que nous n'avons pas connaissance de documents nous permettant de résumer les objectifs liés à l'apprentissage de la planification des cours de la formation pédagogique dispensée dans l'institut de formation que les participant-e-s de la présente étude ont fréquenté. Les documents au niveau intercantonal (CDIP) ne sont pas non plus explicites quant à ce sujet.

3 Les connaissances pédagogiques du contenu consistent en la traduction des connaissances disciplinaires médiatisée par les connaissances pédagogiques générales.

4 L'étude est comprise dans un projet de recherche plus large, portant sur l'évolution des pratiques et croyances des enseignant-e-s. La méthode mixte mobilisée dans ce projet mêle une analyse longitudinale par questionnaires permettant d'explorer tant l'évolution de pratiques d'enseignement, ainsi que des entrevues ouvrant sur les sources de changements et l'articulation entre les facteurs de changement.

5 La durée de la formation varie en fonction du nombre de branches enseignables (une ou deux) choisie(s) par les futurs enseignant-e-s.

6 L'accord inter-juges des Sources des pratiques est compris dans un accord effectué sur l'entier du codage Planification.

7 Les codes inclus dans la catégorie de la planification ont obtenu un accord inter-juges de $71.4 \%$ ( $K=0.68)$ sur 35 unités de sens codées. Les codes relatifs aux sources de changement de pratique et aux types d'apprentissage ont respectivement obtenu un accord inter-juges de $86.7 \%(\mathrm{~K}=0.83)$ et $90 \%$ ( $\mathrm{K}=0.84)$ pour 30 unités de sens.

8 Comme spécifié précédemment, certains cursus proposés à l'IUFE ne comprenaient pas, au moment de l'étude, de cours dédié spécifiquement à la planification des cours. Cette dernière était intégrée aux cours de didactique des branches d'enseignement.

9 Les groupes "pédagogie» et "exploitation du programme» ont été écartés pour des raisons de place limitée. Un choix s'imposant, nous avons opté pour les groupes de pratiques illustrant au mieux l'articulation possible entre pratique - source de changement - type d'apprentissage.

10 Dans le canton de Genève, le collège mène à une maturité gymnasiale. 


\section{Annexe 1}

Guide d'entretien - Planification des cours

- Imaginez que vous devez conseiller un enseignant qui doit planifier un cours dans votre sujet d'expertise pour la toute première fois. Quels conseils en matière de planification lui donneriez-vous?

- En ce qui vous concerne, comment planifiez-vous vos cours?

- Quelle place donnez-vous à la planification dans votre travail?

- Est-ce que vous voyez des spécificités à la planification, dans la branche que vous enseignez?

- Comment planifiez-vous à vos débuts dans l'enseignement?

- Est-ce que vous voyez des éléments particuliers qui auraient joué un rôle dans l' évolution de vos pratique de planification?

- Est-ce que vous avez le sentiment que la formation a joué un rôle dans cette évolution?

- Aujourd'hui, avez-vous le sentiment d'avoir adopté une méthode de travail pour planifier vos cours qui répond à vos objectifs?

- Est-ce que la planification que vous adoptez aujourd'hui nécessite beaucoup d'investissement?

\section{Bibliographie}

Altet, M. (2012). Les compétences de l'enseignant-professionnel: entre savoirs, schèmes d'action et adaptation, le savoir analyser. In L. Paquay, M. Altet, E. Charlier, \& P. Perrenoud (Éd.), Former des enseignants professionnels. Quelles stratégies? Quelles compétences? (4ème éd., pp. 43-57). Bruxelles, Belgique: De Boeck.

Baer, M., Kocher, M., Wyss, C., Guldimann, T., Larcher, S. \& Dörr, G. (2011). Lehrerbildung und Praxiserfahrung im ersten Berufsjahr und ihre Wirkung auf die Unterrichtskompetenzen von Studierenden und jungen Lehrpersonen im Berufseinstieg. Zeitschrift für Erziehungswissenschaften, 14, 85-117.

Blömeke, S., Paine, L., Houang, R., Hsieh, F., Schmidt, W., Han, S., Santillan, M. \& Schwille, J. (2008). Future teachers' competence to plan a lesson: first results of a six-country study on the efficiency of teacher education. ZDM Mathematics Education, 40, 749-762.

Brodhäcker, S. (2014). Unterrichtsplanungskompetenz im Praktikum. Einflussfaktoren auf die Veränderung der wahrgenommenen Kompetenz von Studierenden. Münster, Deutschland: Waxman.

Broeckmans, J. (1986). Short-term developments in student teachers' lesson planning. Teaching and Teacher Education, 2, (3), 215-288.

Charlier, E. (1989). Planifier un cours, c'est prendre des décisions. Bruxelles, Belgique: De Boeck.

Clark, C. M. \& Yinger, R. J. (1979). Three studies of teacher planning (Research Series no. 55). East Lansing, MI: Michigan State University, Institute for Research on Teaching.

Cristol, D. \& Muller, A. (2013). Les apprentissages informels dans la formation pour adultes. Savoirs, 2, (32), 11-59.

Ellis, V. \& Orchard, J. (2014). Learning teaching from experience. Multiple perspectives and international contexts. London, England: Bloomsbury.

Grimmett, P. P. \& MacKinnon, D. P. (1992). Craft knowledge and the education of teachers. Review of Research in Education, 18, 385-456. 
Grossmann, P. (1989). Learning to teach without teacher education. Teachers College Records, 91, (2), 191-208.

Huberman, M. A. (1983). Recipes for busy kitchens: a situational analysis of routine knowledge use in schools. Science Communication, 4, 478-510.

Institut Universitaire de Formation des Enseignants. (2015). Objectifs de la formation et référentiel de compétences relatifs à la formation des enseignantes et enseignants du secondaire I et II. Genève, Suisse: Université de Genève.

Kagan, D. M. \& Tippins, D. J. (1992). The evolution of functional lesson plans among twelve elementary and secondary student teachers. The Elementary School Journal, 92, (4), 477-489.

Lê Van, K. \& Berger, J.-L. (2016, septembre). Evolution of vocational teachers' instructional planning in the early years after teacher education. Poster présenté au 2016 annual meeting of the American Educational Research Association, Washington DC.

Marcel, J.-F., Dupriez, V. \& Périsset Bagnoud, D. (Éd.). (2007). Coordonner, collaborer, coopérer. De nouvelles pratiques enseignantes. Bruxelles, Belgique: De Boeck Université.

Mutton, T., Hagger, H. \& Burn, K. (2011). Learning to plan, planning to learn: the developing expertise of beginning teachers. Teachers and Teaching, 17, (4), 399-416.

Perrenoud, P. (2001). Développer la pratique réflexive dans le métier d'enseignant. Paris, France: ESF.

QSR International Pty Ltd. (2012). NVivo qualitative data analysis software. Version 10 for Windows.

Raynal, F. \& Rieunier, A. (1997). Pédagogie: dictionnaire des concepts clés. Apprentissage, formation, psychologie cognitive. Paris, France: ESF.

Richardson, V. (1996). The role of attitudes and beliefs in learning to teach. In J. Sikula (Éd.), Handbook of research on teacher education (pp. 102-119). New York, NY: Macmillan.

Richardson, V. \& Placier, P. (2001). Teacher change. In V. Richardson (Éd.), Handbook of research on teaching (pp. 905-945). Washington, DC: American Educational Research Association.

Riff, J. \& Durand, M. (1993). Planification et décision chez les enseignants. Bilan à partir des études en éducation physique et sportive, analyses et perspectives. Revue française de pédagogie, 103, 81-107.

Russ, R. S., Sherin, B. L. \& Gamoran Sherin, M. (2016). What constitutes teacher learning? In D. H. Gitomer \& C. A. Bell (Éd.), Handbook of research on teaching (5 ed., pp. 391-438). Washington, DC: American Educational Research Association.

Saldaña, J. (2013). The coding manual for qualitative researchers. Thousand Oaks, CA: Sage.

Schugurensky, D. (2000). The forms of informal learning: towards a conceptualization of the field (WALL Working Paper no. 19). Toronto, ON: Centre for the Study of Education and Work

Shulman, L. S. (1987). Knowledge and teaching: foundations of the new reform. Harvard Educational Review, 57, (1), 1-22.

Simbagoye, A. (2008). Formation pratique et développement professionnel chez les futurs enseignants franco-ontariens: élaboration du savoir-enseigner en contexte de stage. Revue du Nouvel-Ontario, 33, 133-170.

Siwatu, K. O. \& Chesnut, S. R. (2015). The career development of preservice and inservice teachers: why teachers' self-efficacy beliefs matter. In H. Fives \& M. Gregoire Gill (Éd.), International handbook of research on teachers' beliefs (pp. 212-229). New-York, NY: Routledge.

Tochon, F. V. (1993). Le fonctionnement «improvisationnel» de l'enseignant expert. Revue des sciences de l'éducation, 19, (3), 437-461.

Vaudroz, C., Berger, J.-L., Girardet, C. \& Aprea, C. (2015, janvier). Apprendre à préparer ses cours: comment les croyances, connaissances et styles de préparation de cours des enseignants en école professionnelle changent-ils suite à une formation pédagogique? Symposium présenté 
au colloque scientifique international «Condition(s) enseignante(s), Conditions pour enseigner. Réalités, enjeux, défis», Lyon.

Wanlin, P. (2009). La pensée des enseignants lors de la planification de leur enseignement. Revue française de pédagogie, 166, 89-128.

Yildirim, A. (2003). Instructional planning in a centralized school system: lessons of a study among primary school teachers in Turkey. International Review of Education, 49, (5), 525-543.

Zahorik, J. A. (1975). Teachers' planning models. Educational Leadership, 33, 134-139.

Mots-clés: Enseignement, planification, source de changement de pratiques, apprentissage

\section{Unterrichtsplanung erlernen: Entwicklungsverläufe, Quellen und Prozesse des Lernens}

\section{Zusammenfassung}

Unterrichtsplanung ist eine der zentralen Kompetenzen, welche angehende Lehrpersonen während ihrer Ausbildung lernen. Die Quellen und Lernprozesse, welche das Erlernen der Unterrichtsplanung bestimmen, sind noch weitgehend unbekannt. Aufgrund von dreissig halbstrukturierten Interviews mit Absolventinnen und Absolventen des Studiengangs Sekundarstufe zeigt sich, dass diese unterschiedliche Planungsmethoden erlernen und bevorzugen. Zudem wird deutlich, dass die erlernten Unterrichtsplanungen aus verschiedenen Quellen stammen und sich nicht nur auf die in der Ausbildung Vermittelten beschränkte. Weiter waren auch die Prozesse des Lernens unterschiedlich: formal, non-formal und informell. Mit der vorliegenden Studie kann nachgezeichnet werden, dass verschiedene Einflussfaktoren die Entwicklung von Herangehensweisen bei der Unterrichtsplanung beeinflussen.

Schlagworte: Unterrichten, Unterrichtsplanung, Quelle der Veränderung der Praxis, Lernen 


\section{Imparare a pianificare le proprie lezioni: evoluzione, fonti di cambiamento e processi d'apprendimento}

Riassunto

Pianificare le lezioni è una delle competenze fondamentali che gli/le insegnanti sviluppano durante la loro formazione. Le fonti e i processi che stanno alla base dell'apprendimento delle pratiche di pianificazione sono tuttora ampiamente inesplorate. Trenta interviste semi-strutturate condotte presso insegnanti di scuola secondaria che hanno recentemente conseguito il diploma permettono di concludere che il personale insegnante apprende e predilige svariati metodi di pianificazione. Inoltre emerge che tali pratiche provengono da fonti diverse e non si limitano esclusivamente alla formazione: anche i processi di apprendimento sono di vario tipo: formali, non formali e informali. Lo studio mostra pure come i fattori che influiscono sull'evoluzione delle pratiche di pianificazione si articolano fra loro.

Parole chiave: Insegnamento, pianificazione, fonte di cambiamento di pratiche, apprendimento

\section{Learning Instructional Planning: Evolution, Sources of Change, and Learning Processes}

\section{Summary}

Instructional planning is one of the core competencies teachers develop during their training. Sources and processes that shape the learning of instructional planning practices remain largely unexplored. Thirty semi-structured interviews conducted with recently graduated teachers show a panel of instructional planning practices, which are learned and valued by teachers. Moreover, these practices rely on variable sources not limited to teacher education, but also on formal, non-formal, and informal learning. Thus, this study reveals how factors influencing the evolution of instructional practices are articulated.

Keywords: Teaching, planning, source of practice change, learning 
